\title{
Relaxing the Clustering Condition in the Derivation of the KMS Property
}

\author{
Ola Bratteli $\star \star \star$ \\ Centre de Physique Théorique, CNRS Marseille, F-13274 Marseille Cedex 2, France
}

Daniel Kastler

U.E.R. Scientifique de Marseille-Luminy, et Centre de Physique Théorique, CNRS Marseille, F-13274

Marseille Cedex 2, France

\begin{abstract}
We consider as in [1] an infinite dynamical system idealized as a $C^{*}$-algebra acted upon by time-translation automorphisms. We show that a stationary state of such a system which is stable for local perturbations of the dynamics and is clustering in time, either gives rise to a one-sided energy spectrum or is a KMS state. The clustering property assumed here is weaker than the one assumed in [1]. The new proof makes explicit use of spectral properties of clustering states.
\end{abstract}

\section{Introduction}

In an earlier paper, [1], it was proposed to characterize equilibrium states in quantum statistical mechanics by the following three properties

i) Stationarity.

ii) Stability under local perturbations of the dynamics.

iii) Relative purity (clustering).

The system under consideration is idealized as a $C^{*}$-algebra acted upon by a one-parameter group of automorphisms representing the time translations. In [1], it is proved that a state satisfying i), ii), and iii) either gives rise to a one-sided energy spectrum in the corresponding cyclic representation, or satisfies the $\beta$-KMS condition for some inverse temperature $\beta \in \mathbb{R}$. Analogous requirements of stability and clustering applied to the field (rather than to the observable) algebra allow to develop the concept of chemical potential along with that of temperature ([2], announced in [3]).

This paper is devoted to a weakening of the clustering condition iii) of [1]. The resulting new proof is more transparent than the previous one, by making use of additivity properties of the spectra of ergodic groups of transformations.

We next list the definitions and properties needed in this paper. For a more complete discussion of the concepts involved, the reader is referred to [3].

We consider a $C^{*}$-algebra $\mathfrak{A}$, a strongly continuous one-parameter group $t \rightarrow \alpha_{t}$ of *-automorphisms of $\mathfrak{A}$ and a state $\omega$ on $\mathfrak{A} . \omega$ is said to be stationary if it is $\alpha$-invariant.

* Supported by the Norwegian Research Council for Science and Humanities.

$\star \star$ Present address: ZIF der Universität Bielefeld, D-4800 Bielefeld, Federal Republic of Germany. 
(i) $\omega \circ \alpha_{t}=\omega, \quad t \in \mathbb{R}$.

If $\omega$ is stationary there exists a unique strongly continuous unitary group $U(t)$ in the corresponding cyclic representation $\{\pi, \mathscr{H}, \Omega\}$ such that

$$
\begin{aligned}
\pi\left(\alpha_{t}(A)\right) & =U(t) \pi(A) U(t)^{*}, & & t \in \mathbb{R}, A \in \mathfrak{U} \\
U(t) \Omega & =\Omega, & & t \in \mathbb{R} .
\end{aligned}
$$

By Stone's theorem there exists then a self-adjoint operator $H$ such that

$$
U(t)=e^{i t H}, \quad t \in \mathbb{R} .
$$

Now, if $P \in \mathfrak{U}$ is a self-adjoint operator, define a perturbed unitary group by

$$
U^{(P)}(t)=e^{i t(H+P)}
$$

and a perturbed group of automorphisms by

$$
\alpha_{t}^{(P)}(A)=U^{(P)}(t) A U^{(P)}(t)^{*}, \quad t \in \mathbb{R}, A \in \mathfrak{A} .
$$

By a Trotter type argument one can show that $t \rightarrow \alpha_{t}^{(P)}$ is a strongly continuous group of automorphisms of $\mathfrak{U}$, [1]. If $\omega^{(P)}$ is a state of $\mathfrak{U}$ which is $\alpha^{(P)}$ stationary, a straightforward argument by differentiation and integration shows that

$$
\omega^{(P)}\left(\alpha_{T}(A)-\alpha_{S}(A)\right)=i \int_{S}^{T} \omega^{(P)}\left(\left[P, \alpha_{t}(A)\right]\right) d t .
$$

Replacing $P$ by $\lambda P$, dividing by $\lambda$ and using the stationarity of $\omega$, this relation takes the form

$$
\frac{1}{\lambda}\left(\omega^{(\lambda P)}-\omega\right)\left(\alpha_{T}(A)-\alpha_{S}(A)\right)=i \int_{S}^{T} \omega^{(\lambda P)}\left(\left[P, \alpha_{t}(A)\right]\right) d t .
$$

Now, stability can be taken to be any condition ensuring that, as $T \rightarrow \infty, S \rightarrow-\infty$ and $\lambda \rightarrow 0$ in some order, the left hand side tends to zero whilst the right hand side tends to

$$
i \int_{-\infty}^{\infty} \omega\left(\left[P, \alpha_{t}(A)\right]\right) d t
$$

Two such conditions are discussed in [1] and [2]. Araki, [4], has proved that if $\omega$ is a KMS-state for $\alpha$, then $\omega^{(P)}$ exists as a vector-state for all $P=P^{*} \in \mathfrak{U}$, and $\lambda \rightarrow \omega^{(\lambda P)}$ is differentiable for $\lambda=0$ with derivative in $\mathfrak{U}_{*}^{\prime \prime}$. Thus the theorem of this paper is a partial converse of Araki's theorem. As our formulation of stability we take

(ii) for all elements $A, P \in \mathfrak{A}$ such that

$$
\mathbb{R} \ni t \rightarrow \omega\left(\left[P, \alpha_{t}(A)\right]\right)
$$

is an $L^{1}$ function, it is true that

$$
\int_{-\infty}^{\infty} \omega\left(\left[P, \alpha_{t}(A)\right]\right) d t=0 \text {. }
$$


The next condition, hyperclustering of order 4, implies that there is enough such $A, P$ 's in $\mathfrak{A}$. This is a condition of the purity of the state $\omega$.

(iii) There exists a dense subset $\mathfrak{U}_{0}$ of $\mathfrak{U}$, such that for any set $A_{1}, A_{2}, \ldots, A_{p} \in \mathfrak{U}_{0}$, $p \leqq 4$, there are positive constants $C$ and $\delta$ such that

$$
\omega_{(p)}^{T}\left(\alpha_{t_{1}}\left(A_{1}\right), \ldots, \alpha_{t_{p}}\left(A_{p}\right)\right) \leqq C\left\{1+\sup _{i, j}\left|t_{i}-t_{j}\right|\right\}^{-1-\delta},
$$

where $\omega_{(p)}^{T}$ denotes the truncated expectation value of order $p c f$. [3].

By [1], Lemma 3, one can assume that $\mathfrak{A}_{0}$ is closed under regularization by functions $f \in \mathscr{S}(\mathbb{R})$, i.e. if $A \in \mathfrak{A}_{0}$, then $\int_{-\infty}^{\infty} f(t) \alpha_{t}(A) d t \in \mathfrak{A}_{0}$. This will be assumed in the following.

\section{Derivation of the KMS-Condition}

Theorem. Let $\{\mathfrak{U}, \mathbb{R}, \alpha\}$ be a $C^{*}$-dynamical system, and let $\omega$ be a state of $\mathfrak{U}$ which is

(i) Stationary.

(ii) Stable under local perturbations of the dynamics.

(iii) Hyperclustering of order 4.

Then either $\omega$ generates a covariant representation with a one-sided energy spectrum, or $\omega$ is a KMS state for some inverse temperature $\beta \in \mathbb{R}$. (The case of negative or infinite temperature is not ruled out.)

We prove the theorem via some lemmas. For completeness we state all the required lemmas, although some have been proved elsewhere. We assume in the following that $\omega$ satisfies (i), (ii), and (iii).

Let $\{\pi, \mathscr{H}, \Omega\}$ be the cyclic representation of $\mathscr{U}$ generated by $\omega$, define $U$ and $H$ by

$$
\begin{aligned}
& U(t) \pi(A) \Omega=\pi\left(\alpha_{t}(A)\right) \Omega \\
& U(t)=e^{-i t H}, \quad t \in \mathbb{R}, A \in \mathfrak{U} .
\end{aligned}
$$

Let $\mathfrak{M}=\pi(\mathfrak{U})^{\prime \prime}$ and define

$$
\begin{aligned}
& \omega(X)=(\Omega, X \Omega) \\
& \alpha_{t}(X)=U(t) X U(t)^{*}, \quad t \in \mathbb{R}, X \in \mathfrak{M} .
\end{aligned}
$$

Lemma 1. Let $\tau_{t}$ be a weakly continuous group of automorphisms of $\mathfrak{M}$ such that

$$
\begin{aligned}
& \omega \circ \tau_{t}=\omega, \quad t \in \mathbb{R} \\
& \tau_{t} \alpha_{s}=\alpha_{s} \tau_{t}, \quad t, s \in \mathbb{R} .
\end{aligned}
$$

Define the self-adjoint operator $K$ by the requirement

$$
e^{i t K} X \Omega=\tau_{t}(X) \Omega, \quad X \in \mathfrak{M} .
$$

Then $K$ and $H$ commute strongly, and the joint spectrum of $\{H, K\}$ is additive. 
Proof. This is essentially Theorem 4.1 of [3]. The result follows from the clustering properties of $\omega$.

Lemma 2. Either $H$ has one-sided spectrum, or $\operatorname{Sp}(H)=\mathbb{R}$.

Proof. This is the last assertion of Proposition 3 in [1] (for a somewhat better proof see Theorem 4.2 of [3]). The proof makes use of Lemma 1 and of the fact that $\operatorname{Sp}(H)$ has no isolated points (except possibly 0 ) because of the clustering of $\omega$.

For $X, Y \in \mathfrak{M}$, we now define, with $E_{0}=$ the orthogonal projection onto the subspace spanned by $\Omega$.

$$
\left\{\begin{aligned}
F_{X, Y}(t) & =\omega\left(Y \alpha_{t}(X)\right)-\omega(Y) \omega(X) \\
& =\left(Y^{*} \Omega,\left(U(t)-E_{0}\right) X \Omega\right) \\
G_{X, Y}(t) & =\omega\left(\alpha_{t}(X) Y\right)-\omega(X) \omega(Y) \\
& =\left(X^{*} \Omega,\left(U(-t)-E_{0}\right) Y \Omega\right) .
\end{aligned}\right.
$$

For $A, B \in \mathfrak{U}$, set $F_{A, B}=F_{\pi(A), \pi(B)}$ and $G_{A, B}=G_{\pi(A), \pi(B)}$. Then, if $A, B \in \mathfrak{U}_{0}, F_{A, B}$ and $G_{A, B}$ are in $L^{1}$, and by stability

$$
\int F_{A, B}(t) d t=\int G_{A, B}(t) d t .
$$

Using hyperclustering of order 4 one derives from this relation (Proposition 5 and implication (4.5) in [1] or [3], Lemmas 6.3 and 6.4).

Lemma 3. If $A_{1}, B_{1}, A_{2}, B_{2} \in \mathfrak{U}_{0}$, then

$$
\int_{-\infty}^{\infty} F_{A_{1} B_{1}}(t) F_{A_{2} B_{2}}(t) d t=\int_{-\infty}^{\infty} G_{A_{1} B_{1}}(t) G_{A_{2} B_{2}}(t) d t .
$$

From this relation it follows that if $H$ has not one-sided spectrum, then there exists a continuous real function $\Phi$ such that

$$
\Phi(0)=1, \Phi(-E)=\Phi(E)^{-1}, \quad E \in \mathbb{R}
$$

and

$$
\hat{F}_{A, B}(E)=\Phi(E) \hat{G}_{A, B}(E), E \in \mathbb{R}, A, B \in \mathfrak{U}_{0},
$$

where denotes Fourier transform.

In the next lemma, the $\Phi$ in question is the $\Phi$ of Lemma 3 .

Lemma 4. If $H$ has not one-sided spectrum, then $\Omega$ is separating for $\mathfrak{M}$. If $\Delta$ is the modular operator associated to the pair $\{\mathfrak{M}, \Omega\}$, then

$\Phi(-H)=\Delta$.

Proof. Let $H=\int E d P(E)$ be the spectral decomposition of $H$. Then

$$
\begin{aligned}
& F_{X, Y}(t)=\int e^{-i t E}\left(Y^{*} \Omega,\left(d P(E)-d \delta(E) E_{0}\right) X \Omega\right) \\
& G_{X, Y}(t)=\int e^{-i t E}\left(X^{*} \Omega,\left(d P(-E)-d \delta(E) E_{0}\right) Y \Omega\right) .
\end{aligned}
$$

Thus by Lemma 3 , if $X, Y \in \mathfrak{U}_{0}$

$$
\left(Y^{*} \Omega, d P(E) X \Omega\right)=\Phi(E)\left(X^{*} \Omega, d P(-E) Y \Omega\right) .
$$


Now, let $\mathfrak{A}_{00}$ be the subspace of $\mathfrak{A}_{0}$ consisting of elements with bounded Arveson spectrum [3] with respect to $\alpha$. Since $\mathfrak{A}_{0}$ is closed under regularization by functions in $\mathscr{S}, \mathfrak{A}_{00}$ is dense in $\mathfrak{A}$. If $X, Y \in \mathfrak{A}_{00}$, it follows from the relation above that

$$
\left(Y^{*} \Omega, X \Omega\right)=\left(X^{*} \Omega, \Phi(-H) Y \Omega\right)
$$

and since $\Phi(E)>0$ for all $E$,

$$
\left(Y^{*} \Omega, X \Omega\right)=\left(\Phi(-H)^{1 / 2} X^{*} \Omega, \Phi(-H)^{1 / 2} Y \Omega\right) .
$$

Now $\mathfrak{A}_{00}$ is weakly, thus strongly *-dense in $\mathfrak{M}$, [5]. Since $\Phi(-H)^{1 / 2}$ is closed, it follows from (*) that $\mathfrak{M} \Omega \subseteq D\left(\Phi(-H)^{1 / 2}\right)$ and (*) extends to all $X, Y \in \mathfrak{M}$. Now, from $(*)$

$$
X \Omega=0 \Rightarrow X^{*} \Omega=0, \quad X \in \mathfrak{M} .
$$

Hence for $X, Y \in \mathfrak{M}$, since $\mathfrak{M} \Omega$ is dense in $\mathscr{H}$ :

$$
\begin{aligned}
X \Omega=0 & \Rightarrow Y^{*} X \Omega=0 \Rightarrow X^{*} Y \Omega=0 \\
& \Rightarrow X^{*}=0 \Rightarrow X=0 .
\end{aligned}
$$

Thus $\Omega$ is separating for $\mathfrak{M}$, and we may form the modular operator $\Delta$ corresponding to the pair $\{\mathfrak{M}, \Omega\}$. The latter is characterized by

$$
\left(Y^{*} \Omega, X \Omega\right)=\left(\Delta^{1 / 2} X^{*} \Omega, \Delta^{1 / 2} Y \Omega\right), X, Y \in \mathfrak{M} .
$$

By the Tomita theory, $\mathfrak{M} \Omega$ is a core for $\Delta^{1 / 2}$. If $\mathfrak{M}_{M}^{\alpha}$ denotes the Arveson subspace of $\mathfrak{M}$ corresponding to a bounded closed interval $M$, then it is easily seen that $\mathfrak{M}_{M}^{\alpha} \Omega$ is dense in $P(M) \mathscr{H}$, hence $\bigcup_{M} \mathfrak{M}_{M}^{\alpha} \Omega$ is a core for any Borel function of $H$ which is bounded on compacts. Thus $\mathfrak{M} \Omega$ is a core for $\Phi(-H)^{1 / 2}$. It now follows from $(*),(* *)$, and [6], VI, Theorem 2.23 that

$$
\Phi(-H)^{1 / 2}=\Delta^{1 / 2} .
$$

We are now ready to prove the main theorem. Setting $\Delta=e^{K}$, the modular automorphism group $\tau_{t}$ is given by

$$
\tau_{t}(X)=e^{i t K} X e^{-i t K} .
$$

Since $\omega$ is stationary for $\alpha_{t}$, it follows from the uniqueness of the modular automorphism group that

$$
\alpha_{s} \tau_{t}=\tau_{t} \alpha_{s}, \quad t, s \in \mathbb{R} .
$$

Hence by Lemma 1 , the joint spectrum of $(H, K)$ is additive. But by Lemma 4 , this spectrum lies on the curve

$$
\Phi(-h)=e^{k} .
$$

Thus $\log \circ \Phi$ must be a linear function, hence there exists a $\beta \in \mathbb{R}$ such that

$$
K=\beta H .
$$

Thus $\alpha$ satisfies the $\beta$-KMS condition with respect to $\omega$. 
Acknowledgements. One of us (O.B.) is indebted to Derek W. Robinson for discussions and valuable suggestions for improving the proof in this paper.

\section{References}

1. Haag, R., Kastler, D., Trych-Pohlmeyer,E. B.: Stability and equilibrium states. Commun. math. Phys. 38, 173-193 (1974)

2. Haag, R., Kastler, D.: Stability and equilibrium states. II (to appear)

3. Kastler, D.: Equilibrium states of matter and operator algebras. Proc. of the Roma Conf. on $C^{*}$-algebras. Istituto Nationale di Alta Matematica, 1975

4. Araki,H.: Radon Nikodym theorems, relative Hamiltonians, and applications. Varenna Lecture Notes. Lecture at Varenna summer school, Varenna, Italy, 1973, RIMS preprint (1973)

5. Sakai, S.: $C^{*}$-algebras and $W^{*}$-algebras. Berlin-Heidelberg-New York: Springer 1971

6. Kato,T.: Perturbation theory for linear operators. Berlin-Heidelberg-New York: Springer 1966

Communicated by J.L. Lebowitz

Received July 26, 1975

Note Added in Proof. After finishing this paper we have been made aware of that the main theorem has been proved independently by Frank Hoekman. 